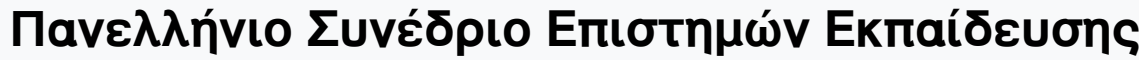

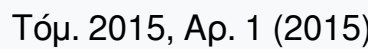

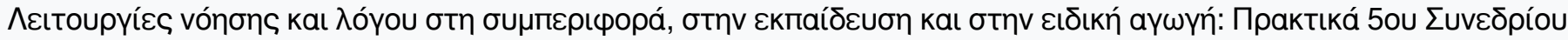

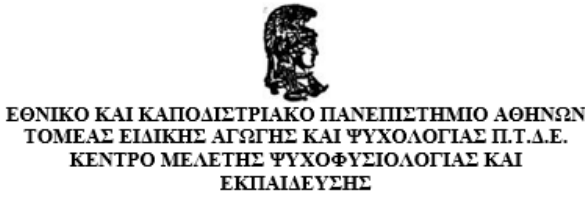

$5^{\circ}$ IANEA AHNIO IYNEAPIO

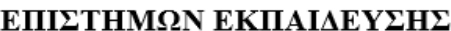

19-21 Iovvíov 2015

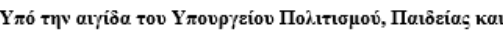

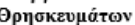

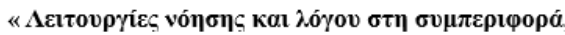

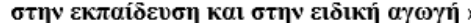

\section{IPAKTIKA ГYNEAPIOY}

\author{
AIIPIAIOE 2016 \\ EIIMEAEIA:

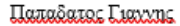

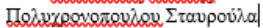

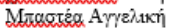 \\ ISSN: 2529-1157
}

AOHNA

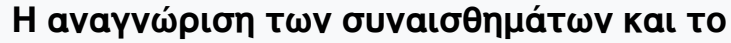

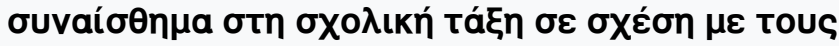

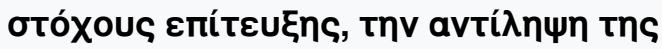

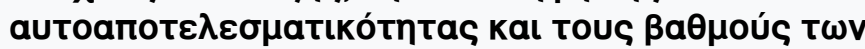

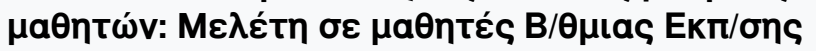

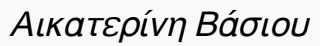

doi: $10.12681 /$ edusc. 148

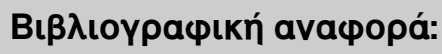

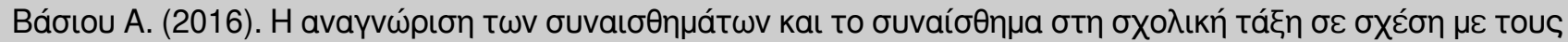

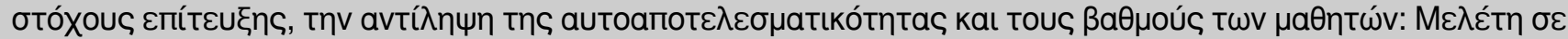

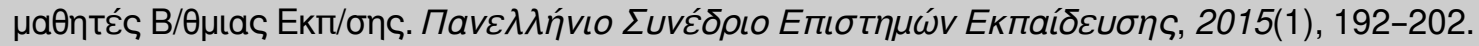
https://doi.org/10.12681/edusc.148 


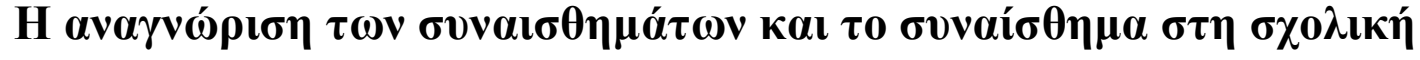

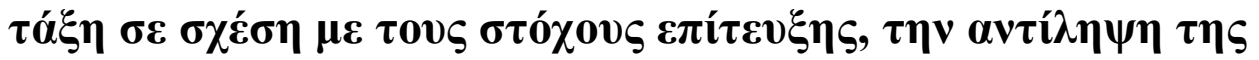

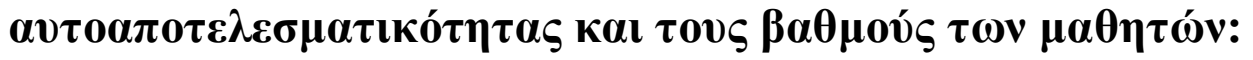

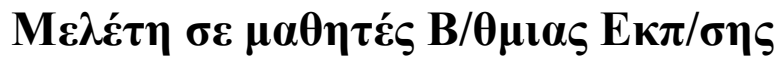

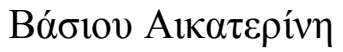

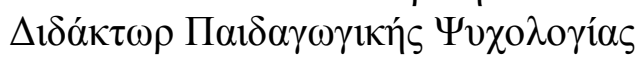 \\ ekvasiou@uth.gr
}

\begin{abstract}
Пері́ $\lambda \psi \eta$

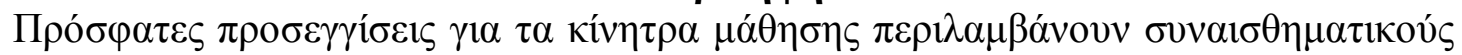

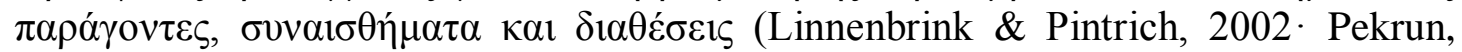

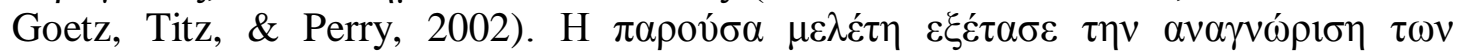

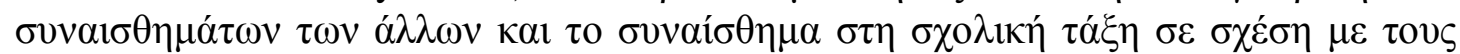

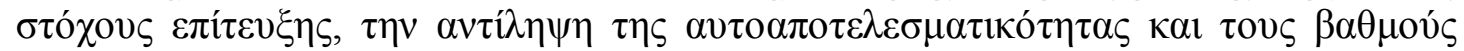

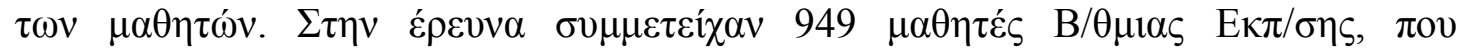

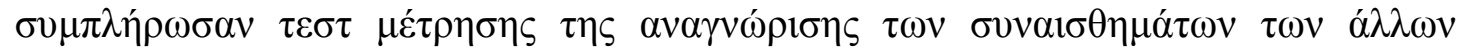

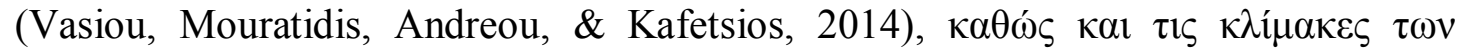

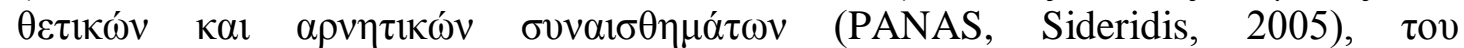

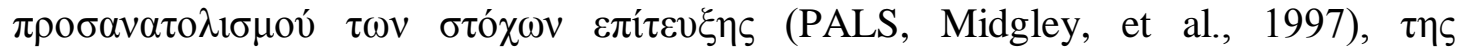

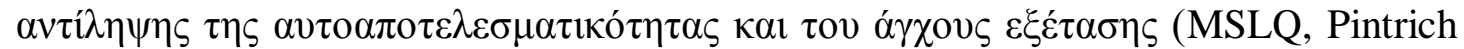

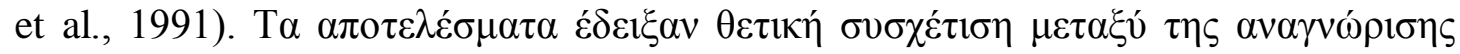

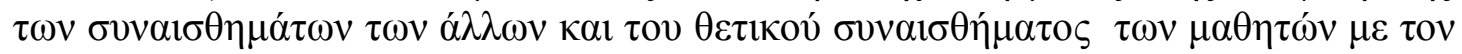

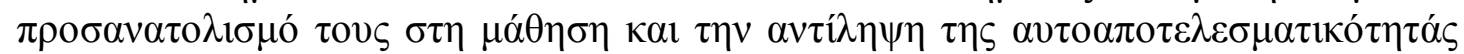

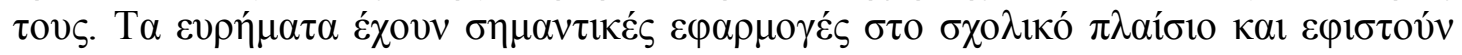

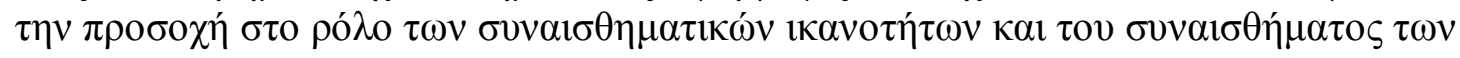

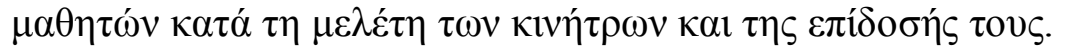

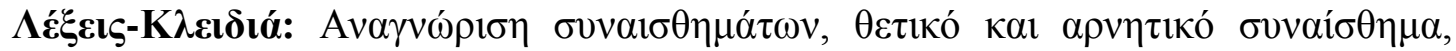

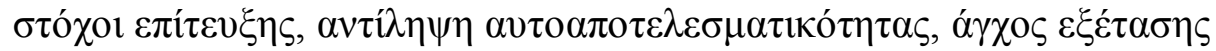

\begin{abstract}
Recent approaches about achievement goals include emotional factors, emotions and moods (Linnenbrink \& Pintrich, 2002 · Pekrun, Goetz, Titz, \& Perry, 2002). The present study examined the emotion perception and emotion in the classroom in relation to the achievement goals, self-efficacy and students' grades. In research participated 949 Secondary education students, who completed a test of emotion perception (Vasiou, Mouratidis, Andreou, \& Kafetsios, 2014), and the scales of positive and negative emotions (PANAS, Sideridis, 2005), goals orientation (PALS, Midgley, et al., 1997), self-efficacy and test anxiety (MSLQ, Pintrich et al., 1991). The findings have important applications in schools and draw attention to the role of emotional abilities and students' emotions in the study of students' motivation and performance.
\end{abstract}


Keywords: Emotion perception, positive and negative affect, achievement goals, selfefficacy, test anxiety

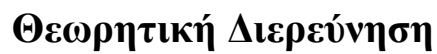

\section{O $\pi \rho 0 \sigma \alpha v \alpha \tau \sigma \lambda \iota \sigma \mu o ́ \varsigma \tau \omega v \sigma \tau o ́ \chi \omega v ~ \varepsilon \pi i ́ \tau \varepsilon v \xi \eta \varsigma \tau \omega v \mu \alpha \theta \eta \tau \omega ́ v$}

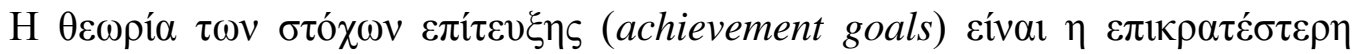

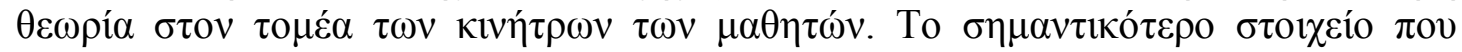

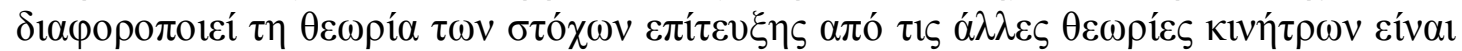

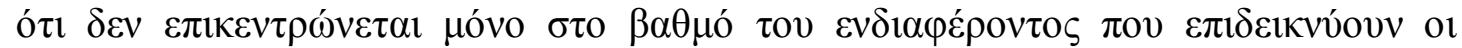

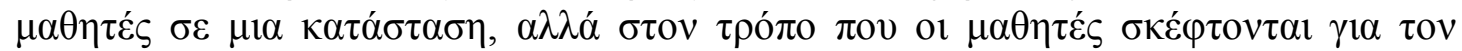

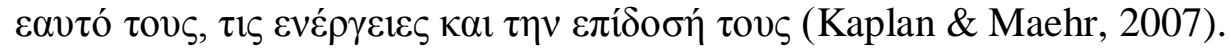

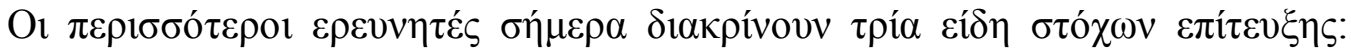

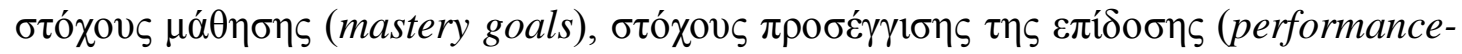

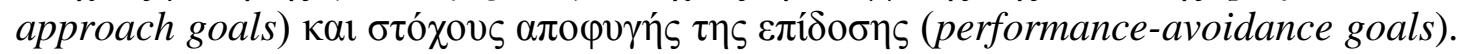

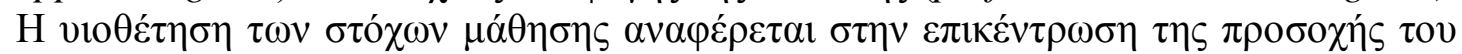

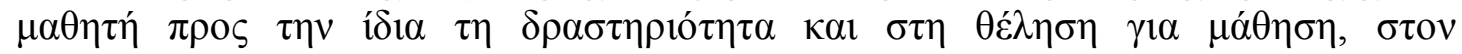

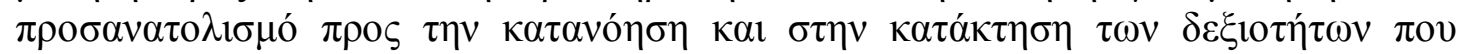

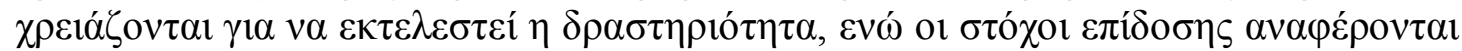

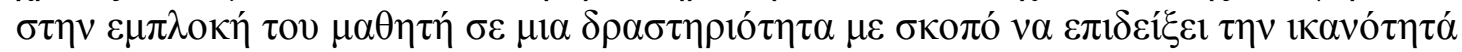

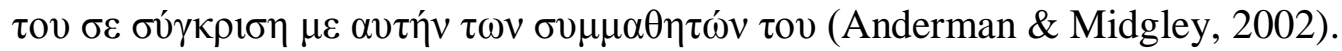

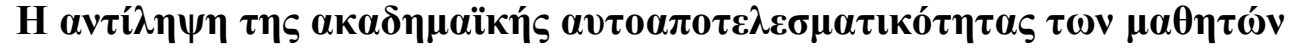

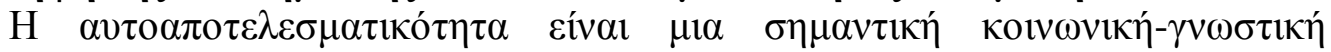

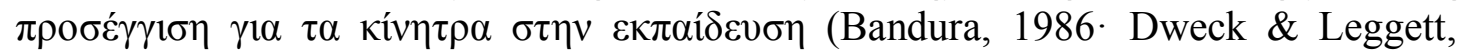

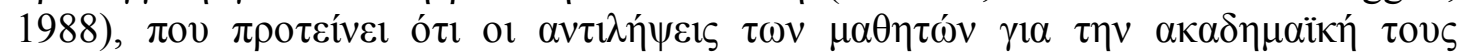

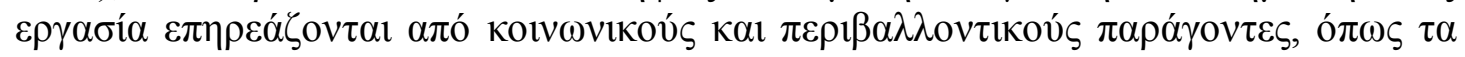

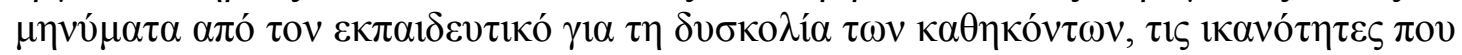

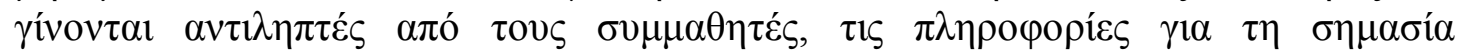

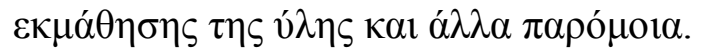

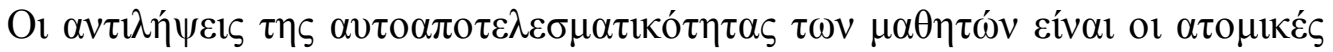

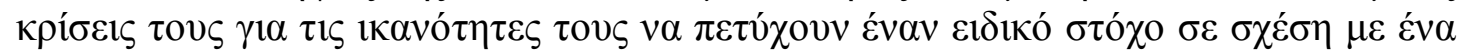

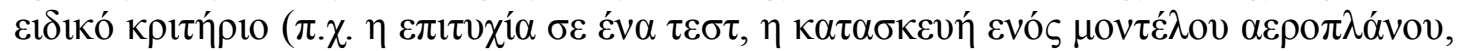

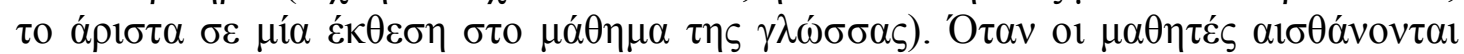

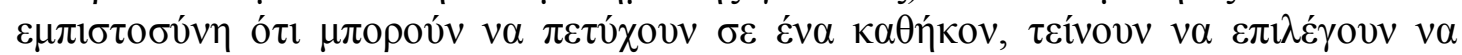

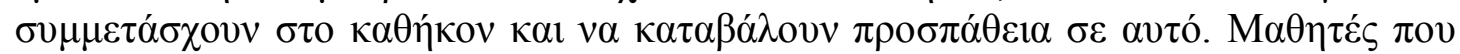

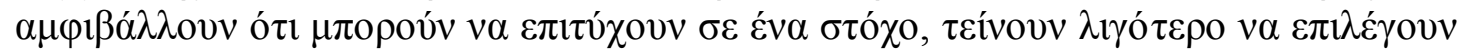

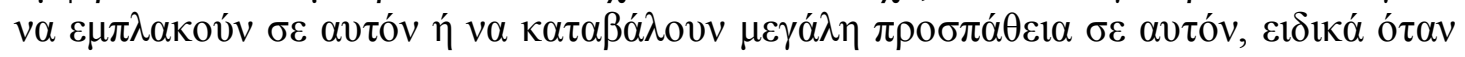

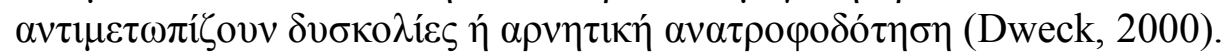

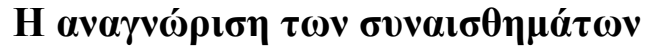

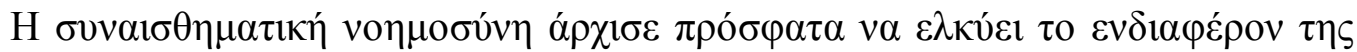
$\varepsilon \kappa \pi \alpha \iota \delta \varepsilon v \tau \iota \kappa \eta ́ \varsigma ~ \varepsilon ́ \rho \varepsilon v v \alpha \varsigma$ (Ciarrochi, Deane, \& Anderson, 2002- Mayer, Salovey, \&

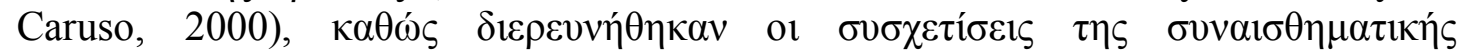

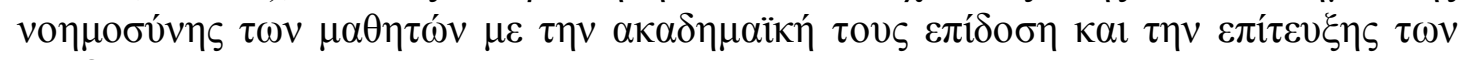

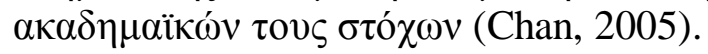

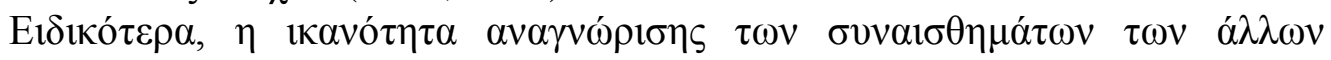

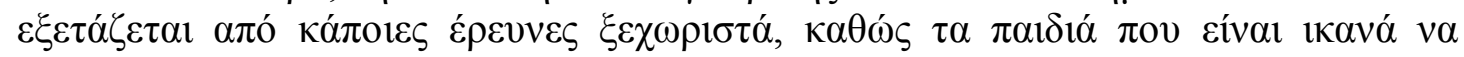




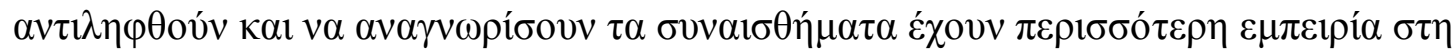

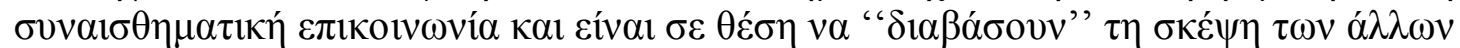

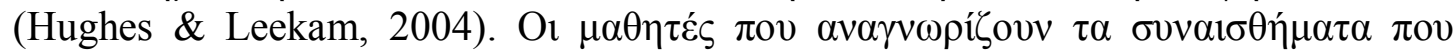
$\varepsilon \kappa \varphi \rho \alpha ́ \zeta o v \tau \alpha \imath$ a

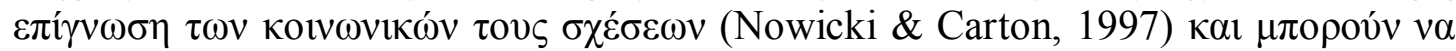

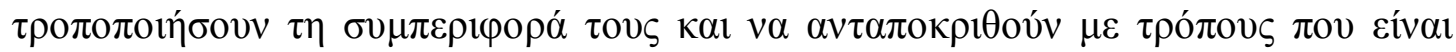

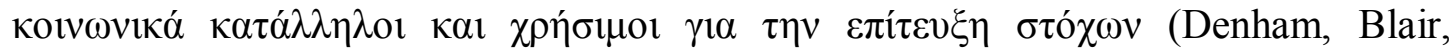
DeMulder, Levitas, Sawyer, Auerbach-Major, \& Queenan, 2003· Ekman, 2003), عvó

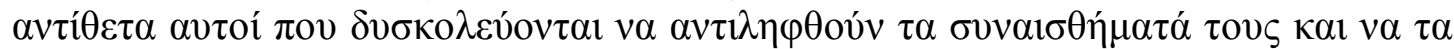

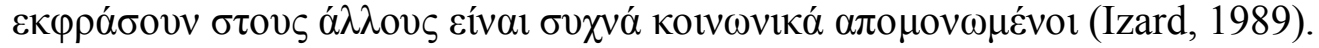

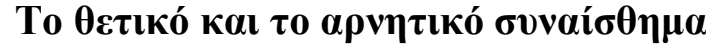

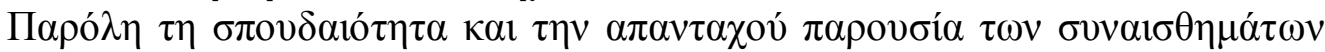

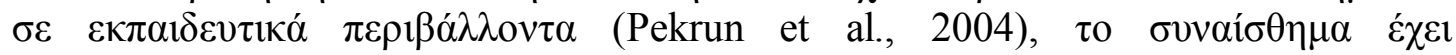

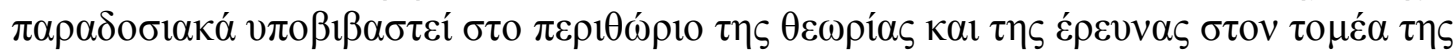

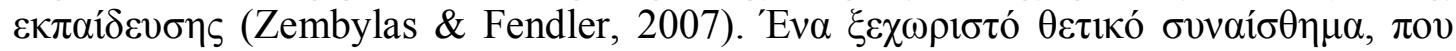

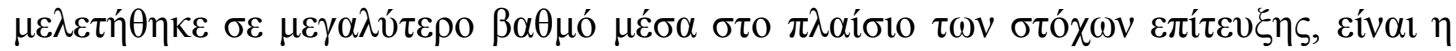

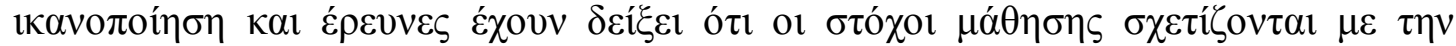

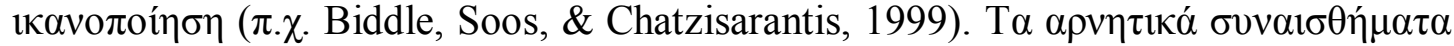

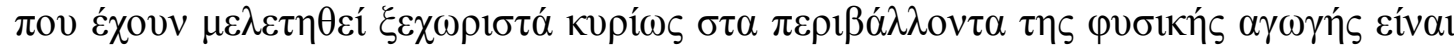

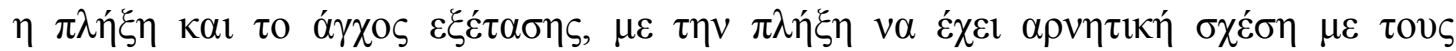

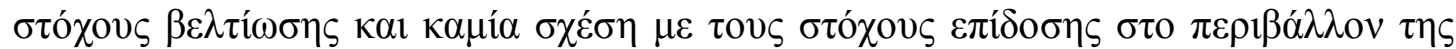

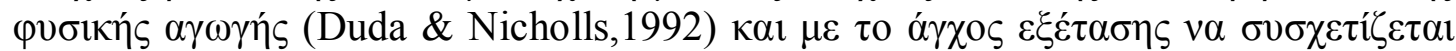

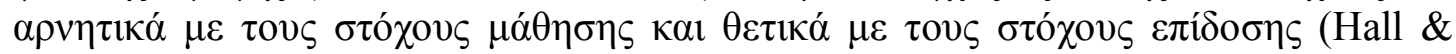

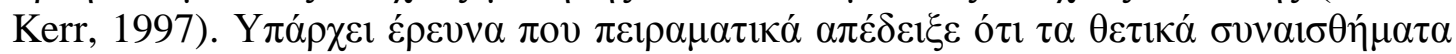

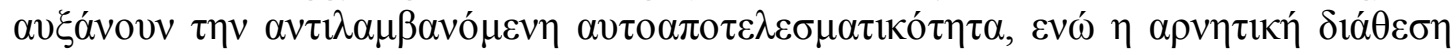
$\tau \eta v \mu \varepsilon ı \omega ́ v \varepsilon ı$ (Kavanaugh \& Bower, 1985).

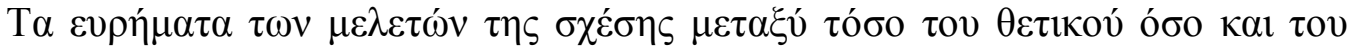

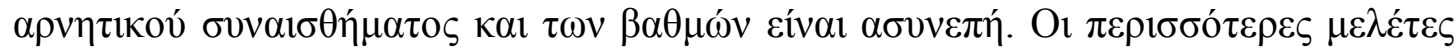

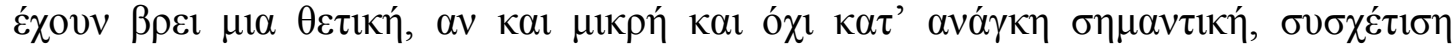

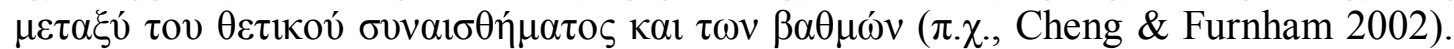

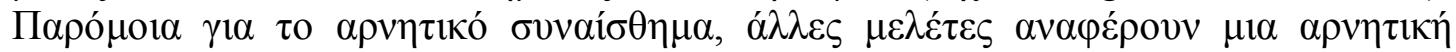

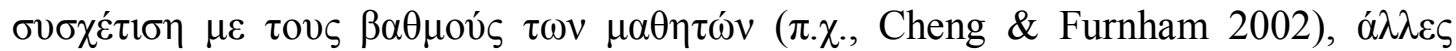

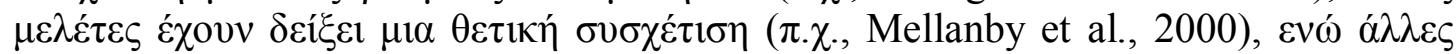

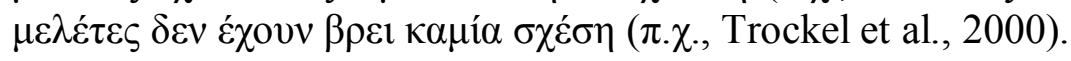

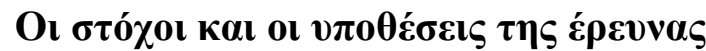

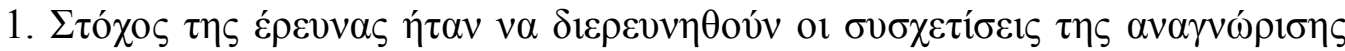

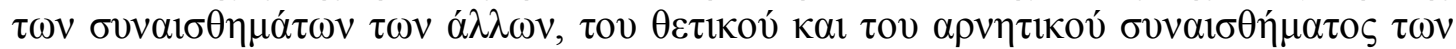

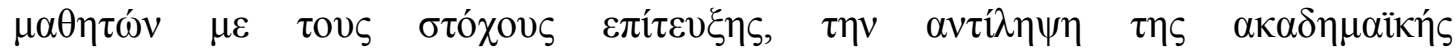

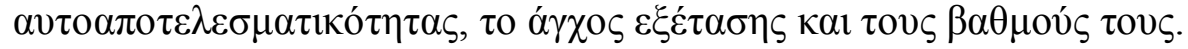

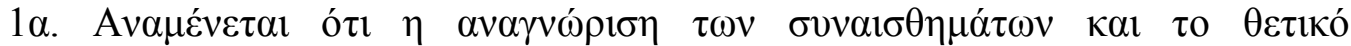

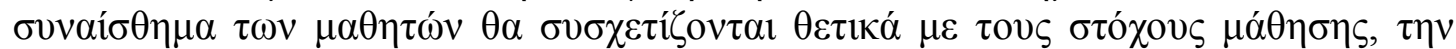

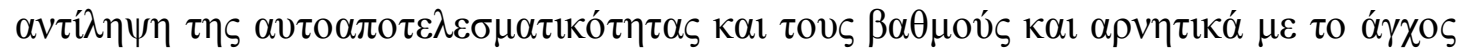
$\varepsilon \xi \dot{\varepsilon} \tau \alpha \sigma \eta \zeta$.

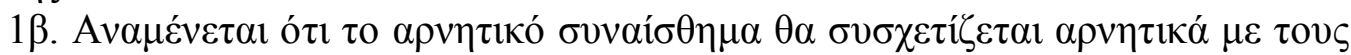

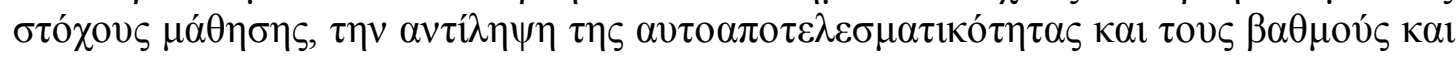

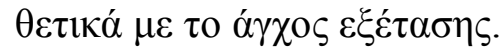




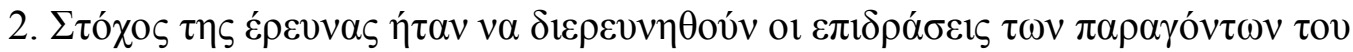

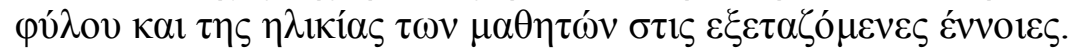

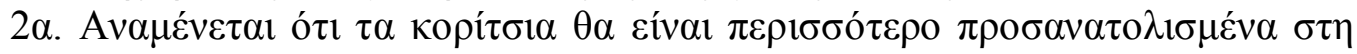

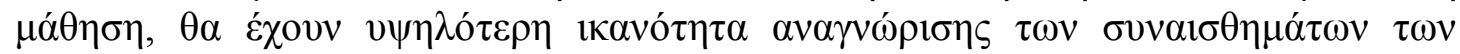

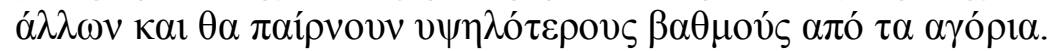

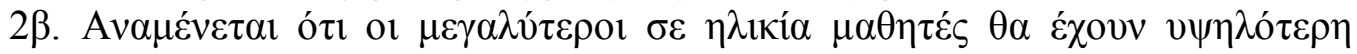

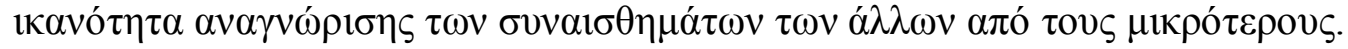

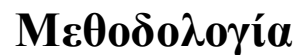

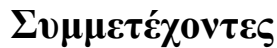

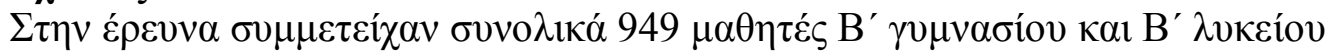

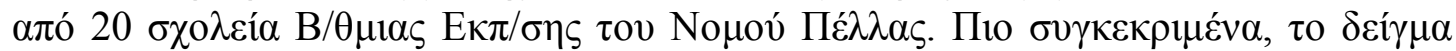

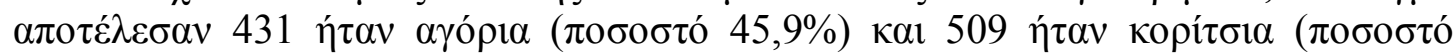

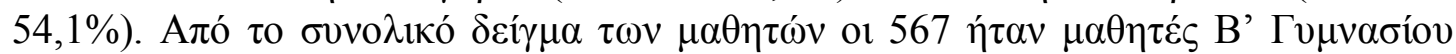
(

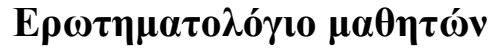

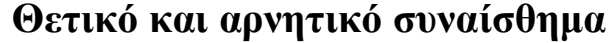

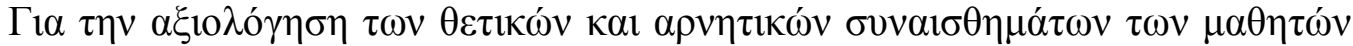

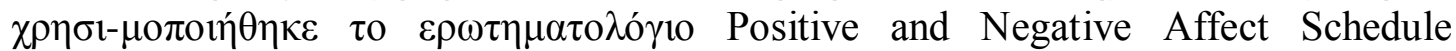

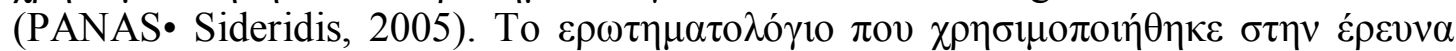

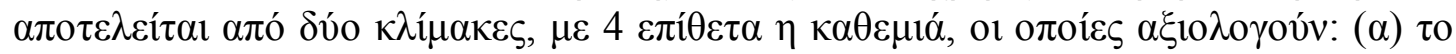

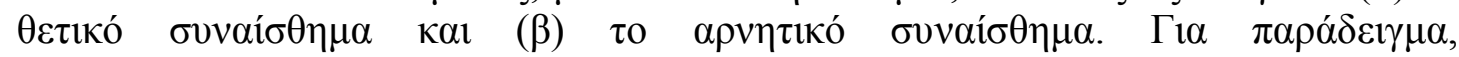

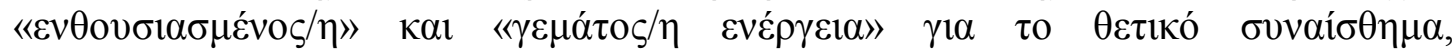

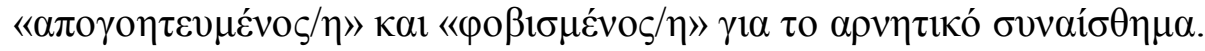

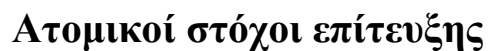

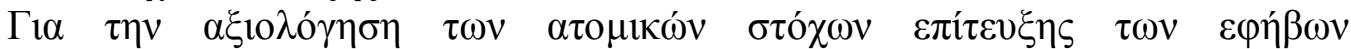

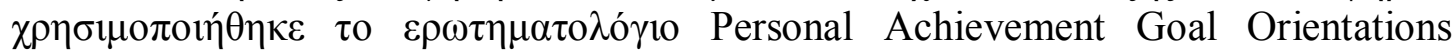
(PALS• Midgley, Maehr, Hicks, Roeser, Urdan, Anderman, Kaplan, Arunkumar \&

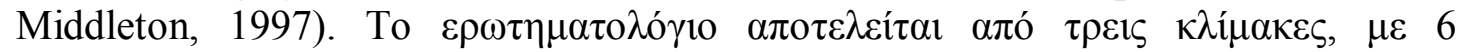

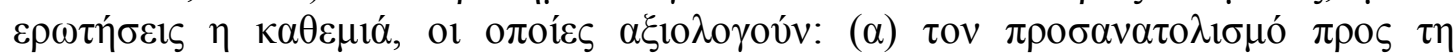

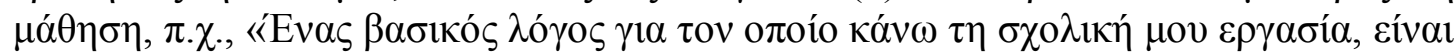

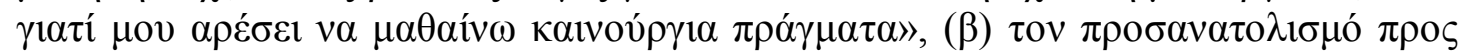

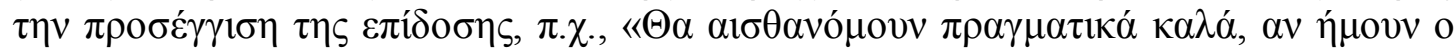

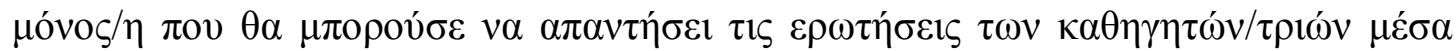

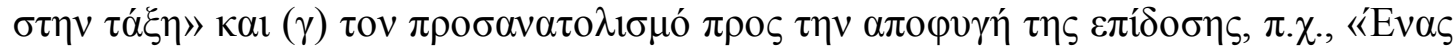

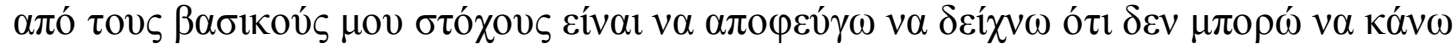

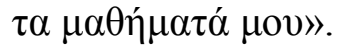

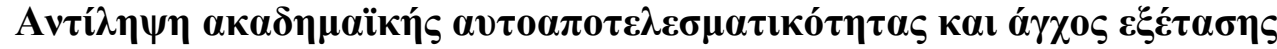

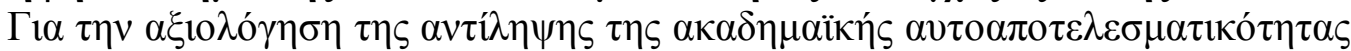

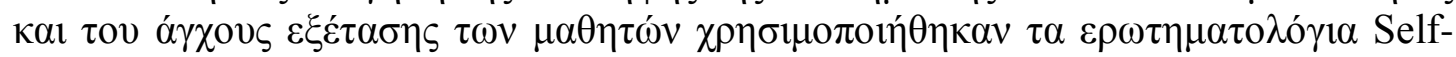

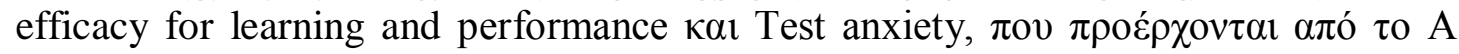
manual for the Use of the Motivation Strategies for Learning Questionnaire (MSLQ)

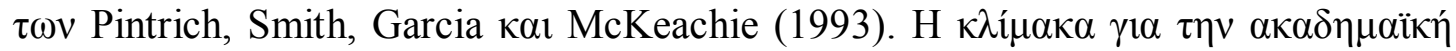




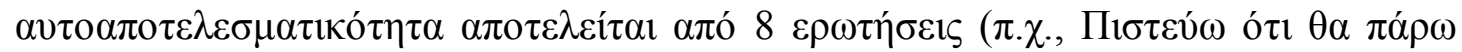

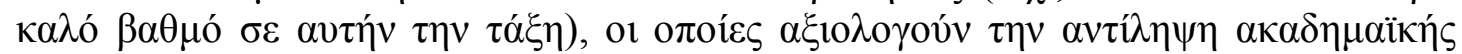

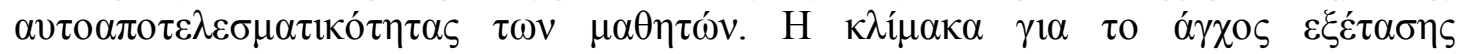

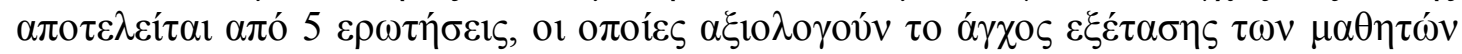

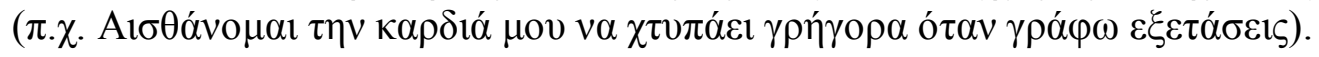

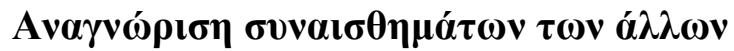

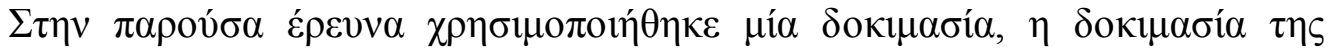

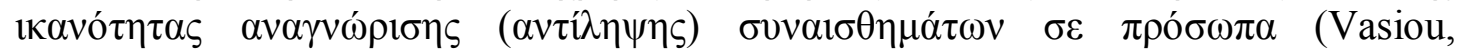
Mouratidis, Andreou, \& Kafetsios, 2014). O1 $\mu \alpha \theta \eta \tau \dot{\varepsilon} \varsigma \kappa \lambda \eta \dot{\theta \eta \kappa \alpha \nu ~ v \alpha ~ \alpha v \alpha \gamma v \omega \rho i ́ \sigma o v v ~} 6$

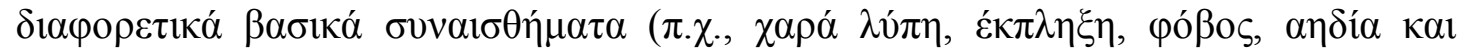

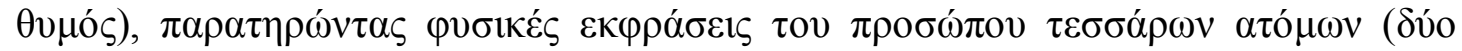

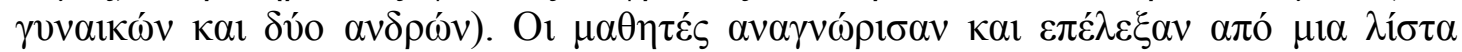

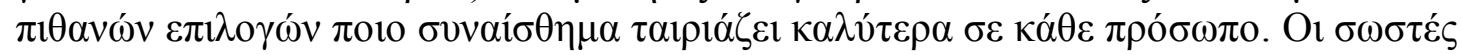

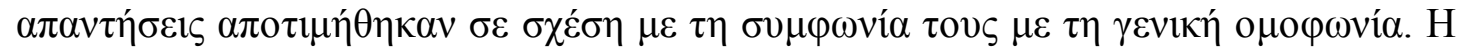

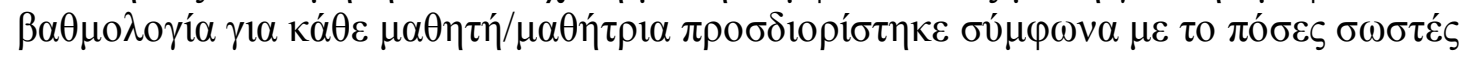
$\alpha \pi \alpha \nu \tau \eta ́ \sigma \varepsilon ı \varsigma \varsigma ~ \varepsilon ́ \delta \omega \sigma \varepsilon$.

\section{Bä $\boldsymbol{\mu} \boldsymbol{\alpha o ́}$}

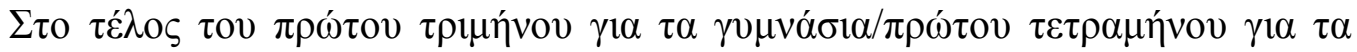

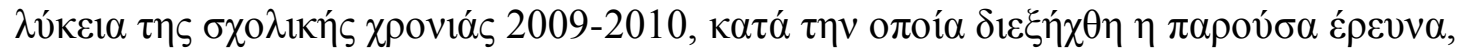

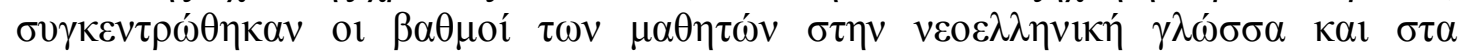

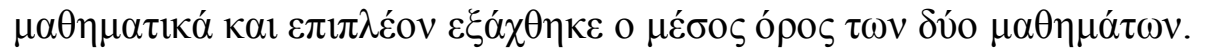

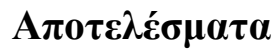

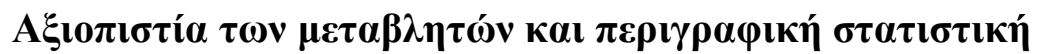

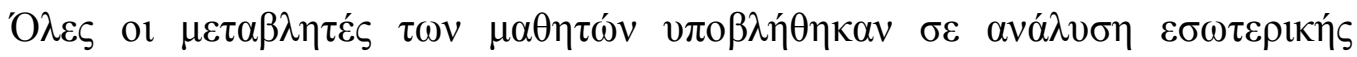

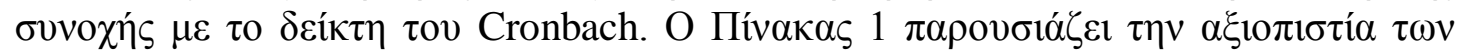

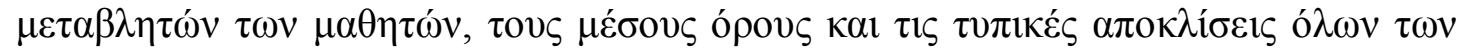

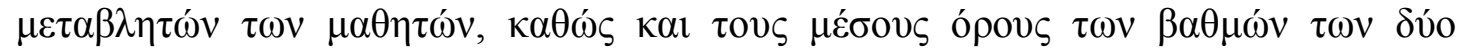

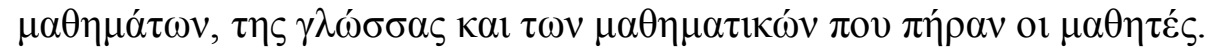

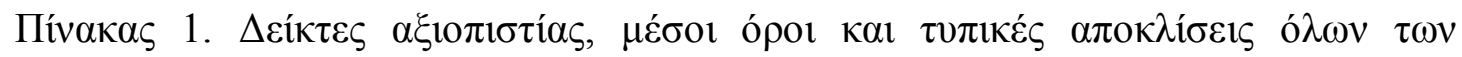
$\mu \varepsilon \tau \alpha \beta \lambda \eta \tau \omega ́ v \tau \omega \nu \mu \alpha \theta \eta \tau \omega ́ v$

\begin{tabular}{|c|c|c|c|}
\hline $\begin{array}{l}\text { METAB } \Lambda \text { HE } \Sigma \\
\mathrm{N}=949\end{array}$ & $\begin{array}{l}\Delta . A . \\
\alpha>0,5\end{array}$ & M.O. & T.A. \\
\hline 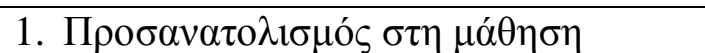 & $\alpha=0,83$ & 3,05 & 0,96 \\
\hline 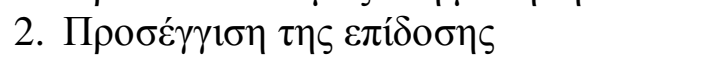 & $\alpha=0,75$ & 2,86 & 1,02 \\
\hline 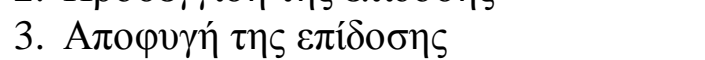 & $\alpha=0,69$ & 2,69 & 0,94 \\
\hline 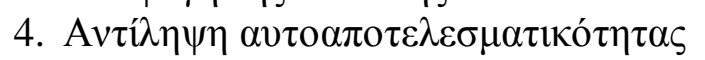 & $\alpha=0,83$ & 4,96 & 1,09 \\
\hline 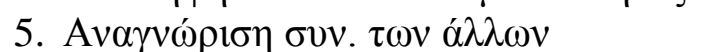 & ------ & 0,73 & 0,13 \\
\hline 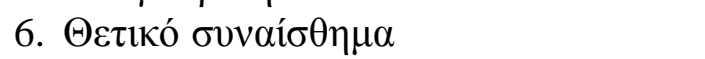 & $\alpha=0,60$ & 3,42 & 0,86 \\
\hline 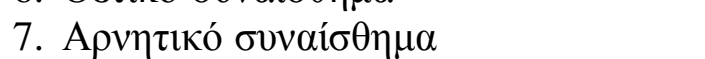 & $\alpha=0,62$ & 1,40 & 0,57 \\
\hline 8. А $\gamma \chi \circ \varsigma \varepsilon \xi \dot{\varepsilon} \tau \alpha \sigma \eta \varsigma$ & $\alpha=0,76$ & 3,91 & 1,50 \\
\hline 9. В $\alpha \theta \mu$ оí ( $\gamma \lambda \omega ́ \sigma \sigma \alpha \varsigma \& ~ \mu \alpha \theta \eta \mu \alpha \tau \imath \kappa \omega ́ v)$ & ------ & 15,21 & 2,83 \\
\hline
\end{tabular}




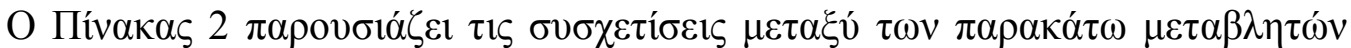

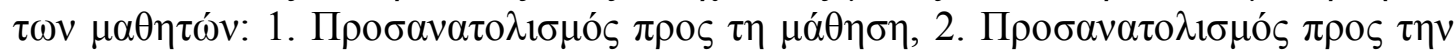

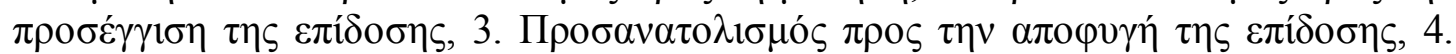

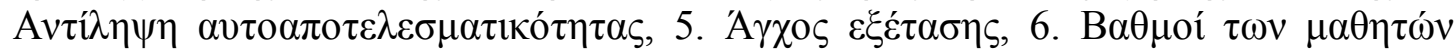

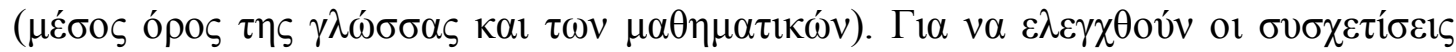

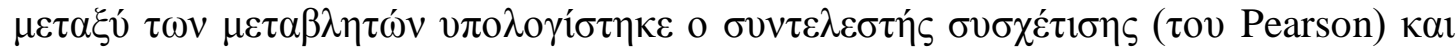

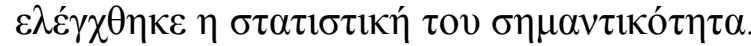

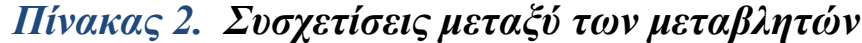

\begin{tabular}{|c|c|c|c|c|c|c|}
\hline $\begin{array}{l}\text { METAB } \Lambda \text { HTE } \\
\text { N=949 }\end{array}$ & 1 & 2 & 3 & 4 & 5 & 6 \\
\hline 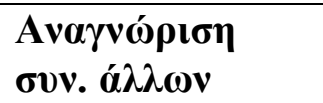 & $\mathbf{0 , 0 9 * *}$ & $-0,03$ & $-0,05$ & $0,13 * *$ & $-0,03$ & $0,12 * *$ \\
\hline 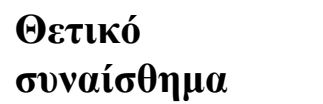 & $0,25 * *$ & $0,22 * *$ & $\mathbf{0 , 0 8 *}$ & $\mathbf{0 , 3 1} * *$ & $-0,07 *$ & 0,06 \\
\hline 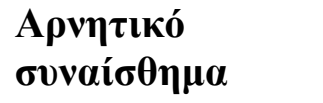 & $-0,09 *$ & 0,02 & $0,08 *$ & $-0,11 * *$ & $\mathbf{0 , 1 9} * *$ & $-0,08^{*}$ \\
\hline
\end{tabular}

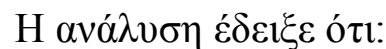

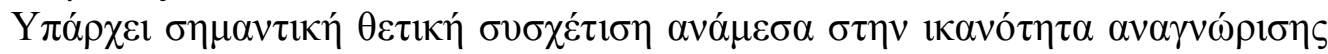

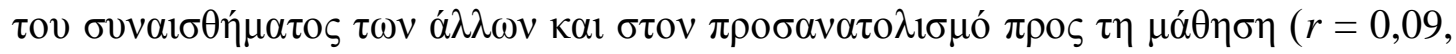

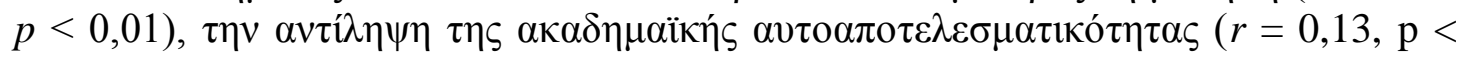

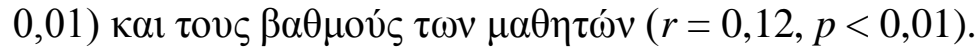

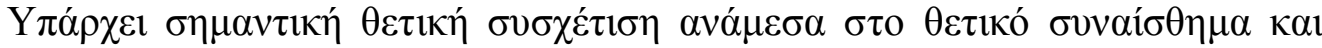

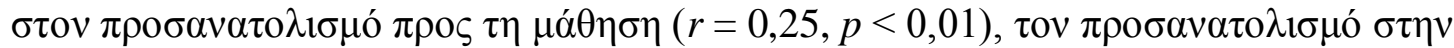

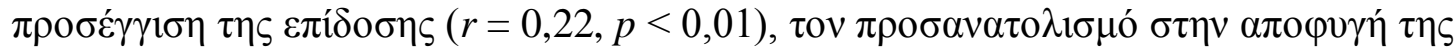

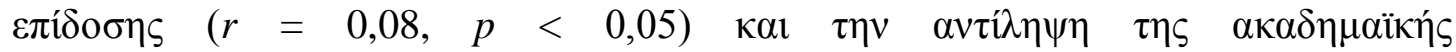

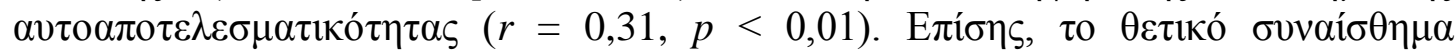

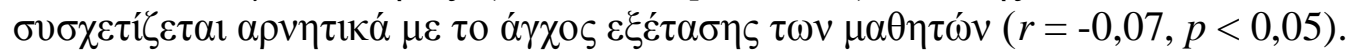

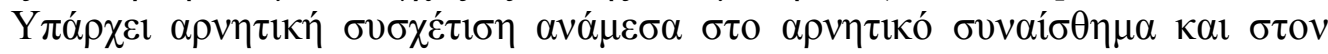

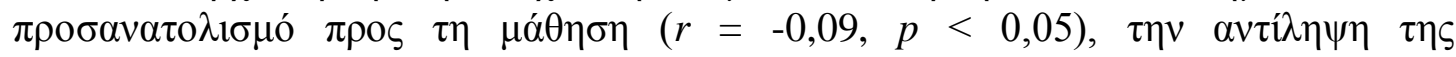

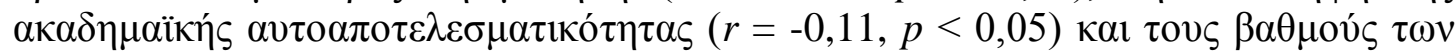

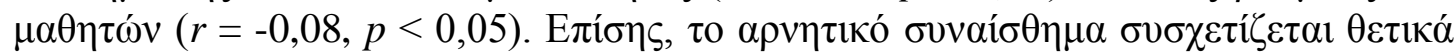

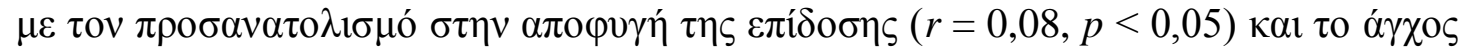
$\varepsilon \xi \dot{\varepsilon} \tau \alpha \sigma \eta \varsigma \tau \omega \nu \mu \alpha \theta \eta \tau \omega ́ v(r=0,19, p<0,01)$.

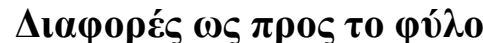

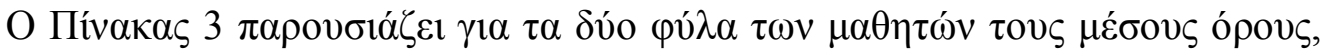

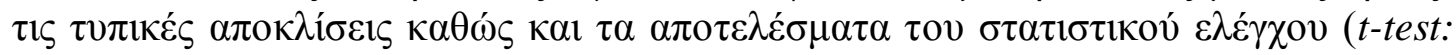
independent samples).

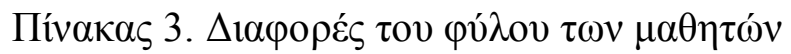

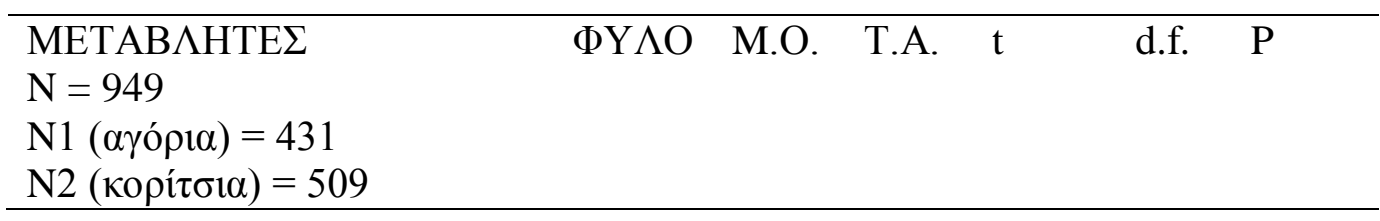




\begin{tabular}{|c|c|c|c|c|c|c|c|}
\hline \multirow[t]{2}{*}{1.} & \multirow{2}{*}{ 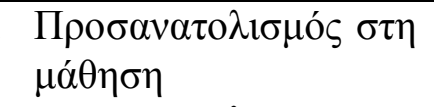 } & 1 & 2,93 & 1,02 & $-3,63$ & 938 & $\overline{0,000}$ \\
\hline & & 2 & 3,16 & 0,91 & & & \\
\hline & 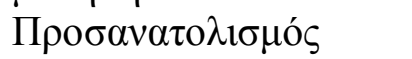 & 1 & 2,92 & 0,96 & 1,42 & 938 & 0,155 \\
\hline & 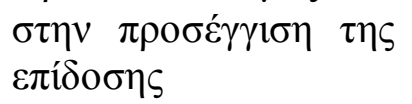 & 2 & 2,83 & 1,07 & & & \\
\hline \multirow[t]{2}{*}{3.} & 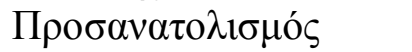 & 1 & 2,70 & 0,95 & 0,25 & 938 & 0,805 \\
\hline & $\begin{array}{l}\sigma \tau \eta \nu \quad \alpha \pi \circ \varphi v \gamma \eta \dot{ } \quad \tau \eta \varsigma \\
\varepsilon \pi i ́ \delta \circ \sigma\rceil \varsigma\end{array}$ & 2 & 2,68 & 0,93 & & & \\
\hline & 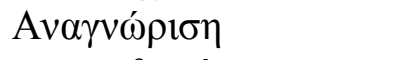 & 1 & 0,71 & 0,13 & $-5,67$ & 938 & $\mathbf{0 , 0 0 0}$ \\
\hline & 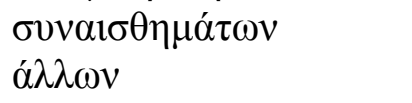 & 2 & $\mathbf{0 , 7 5}$ & 0,12 & & & \\
\hline \multirow[t]{2}{*}{5.} & $A \nu \tau i ́ \lambda \eta \psi \eta$ & 1 & 4,96 & 1,11 & $-0,10$ & 938 & 0,922 \\
\hline & 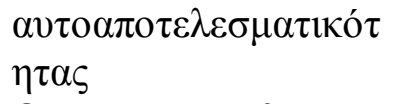 & 2 & 4,97 & 1,08 & & & \\
\hline \multirow[t]{2}{*}{6.} & 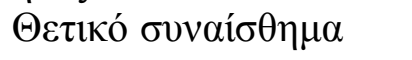 & 1 & 3,56 & 0,80 & 4,54 & 938 & 0,000 \\
\hline & & 2 & 3,31 & 0,89 & & & \\
\hline \multirow[t]{2}{*}{7.} & 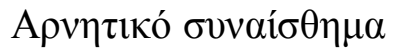 & 1 & 1,36 & 0,56 & $-1,99$ & 938 & 0,047 \\
\hline & & 2 & 1,44 & 0,58 & & & \\
\hline \multirow[t]{2}{*}{8 . } & A $\gamma \chi \propto \varsigma$ $\varepsilon \xi \dot{\varepsilon} \tau \alpha \sigma \eta \varsigma$ & 1 & 3,74 & 1,49 & $-3,31$ & 938 & 0,001 \\
\hline & & 2 & 4,06 & 1,51 & & & \\
\hline \multirow[t]{2}{*}{9.} & B $\alpha \theta \mu$ oí ( & 1 & 14,65 & 2,89 & $-5,58$ & 938 & 0,000 \\
\hline & $\begin{array}{l}\gamma \lambda \omega ́ \sigma \sigma \alpha \varsigma \\
\mu \alpha \theta \eta \mu \alpha \kappa \omega ́ v)\end{array}$ & 2 & 15,68 & 2,70 & & & \\
\hline
\end{tabular}

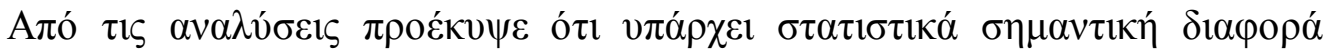

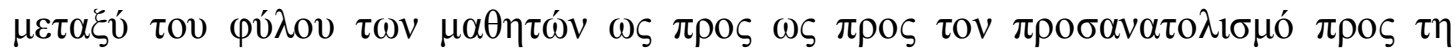

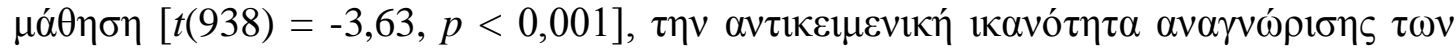

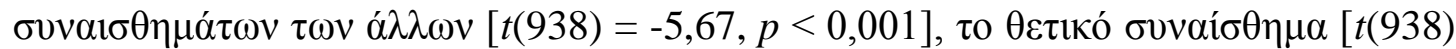

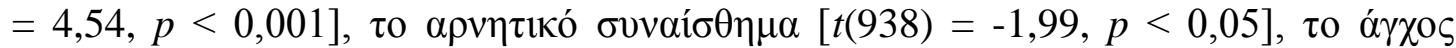

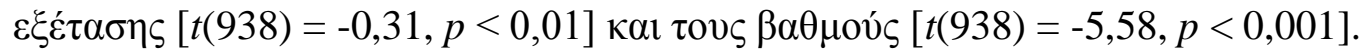

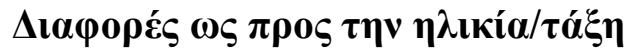

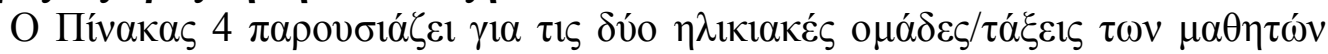

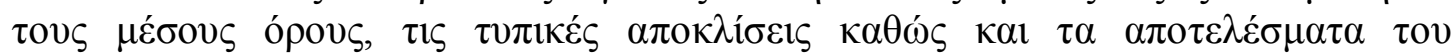
$\sigma \tau \alpha \tau \imath \sigma \tau 1 \kappa о v ́ ~ \varepsilon \lambda \varepsilon ́ \gamma \gamma \chi(t$-test: independent samples).

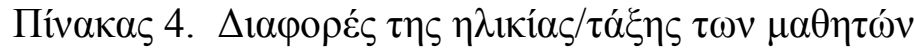

\begin{tabular}{|c|c|c|c|c|c|c|}
\hline 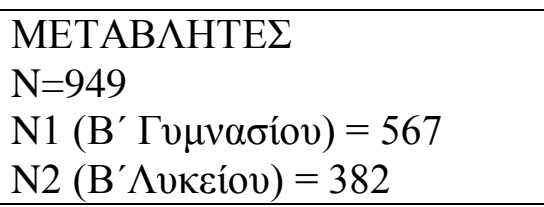 & 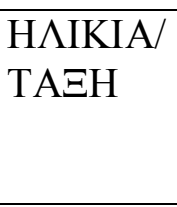 & M.O. & T.A. & $\mathrm{t}$ & d.f. & $\mathrm{P}$ \\
\hline 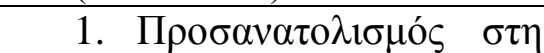 & 1 & 2,98 & 0,97 & $-2,63$ & 947 & 0,007 \\
\hline$\mu \alpha ́ \theta \eta \sigma \eta$ & 2 & 3,15 & 0,95 & & & \\
\hline 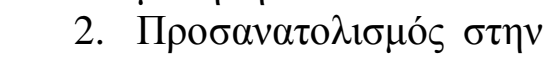 & 1 & 3,07 & 1,02 & 7,72 & 947 & $\mathbf{0 , 0 0 0}$ \\
\hline $\begin{array}{l}\pi \rho 0 \sigma \varepsilon ́ \gamma \gamma 1 \sigma \eta \\
\varepsilon \pi i ́ \delta \circ \sigma \eta \varsigma\end{array}$ & 2 & 2,56 & 0,95 & & & \\
\hline 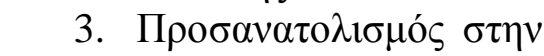 & 1 & 2,93 & 0,90 & 9,94 & 947 & $\mathbf{0 , 0 0 0}$ \\
\hline 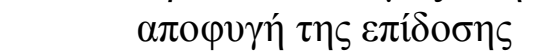 & 2 & 2,34 & 0,89 & & & \\
\hline 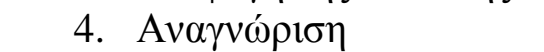 & 1 & 0,71 & 0,14 & $-4,36$ & 947 & $\mathbf{0 , 0 0 0}$ \\
\hline
\end{tabular}




\begin{tabular}{|c|c|c|c|c|c|c|c|}
\hline & $\sigma v v \alpha 1 \sigma \theta \eta \mu \alpha ́ \tau \omega \nu$ ó $\lambda \lambda \omega \nu$ & 2 & $\mathbf{0 , 7 5}$ & 0,11 & & & \\
\hline \multirow[t]{3}{*}{5.} & $A \nu \tau i ́ \lambda \eta \psi \eta$ & 1 & 5,05 & 1,09 & 3,05 & 947 & 0,002 \\
\hline & $\alpha v \tau \mathrm{O} \alpha \pi \mathrm{\tau} \tau \varepsilon \lambda \varepsilon \sigma \mu \alpha \tau \iota \kappa o ́ \tau \eta$ & 2 & 4,83 & 1,10 & & & \\
\hline & $\tau \alpha \varsigma$ & & & & & & \\
\hline \multirow{2}{*}{6.} & 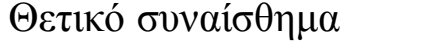 & 1 & 3,56 & 0,85 & 6,23 & 947 & $\mathbf{0 , 0 0 0}$ \\
\hline & & 2 & 3,21 & 0,83 & & & \\
\hline \multirow[t]{2}{*}{7.} & 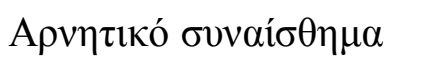 & 1 & 1,33 & 0,51 & $-4,69$ & 947 & $\mathbf{0 , 0 0 0}$ \\
\hline & & 2 & $\mathbf{1 , 5 1}$ & 0,65 & & & \\
\hline \multirow[t]{2}{*}{8.} & A $\gamma \chi 0 \varsigma$ $\varepsilon \xi \dot{\varepsilon} \tau \alpha \sigma \eta \varsigma$ & 1 & 4,02 & 1,48 & 2,77 & 947 & 0,006 \\
\hline & & 2 & 3,75 & 1,54 & & & \\
\hline \multirow[t]{2}{*}{9.} & 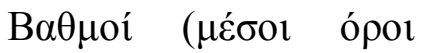 & 1 & 15,21 & 2,95 & 0,01 & 947 & 0,989 \\
\hline & $\begin{array}{l}\gamma \lambda \omega ́ \sigma \sigma \alpha \varsigma \\
\mu \alpha \theta \eta \mu \alpha \tau 1 \kappa \omega ́ v)\end{array}$ & 2 & 15,21 & 2,65 & & & \\
\hline
\end{tabular}

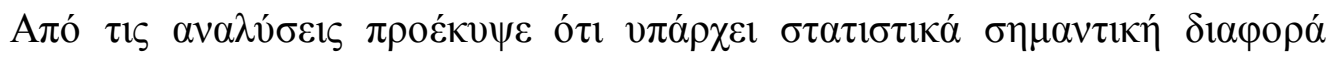

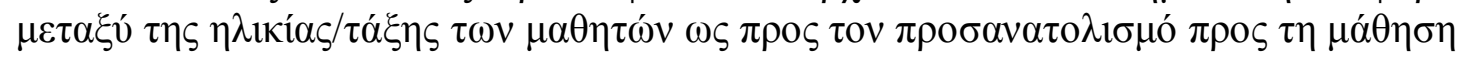

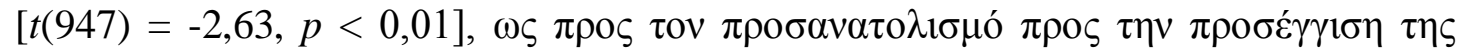

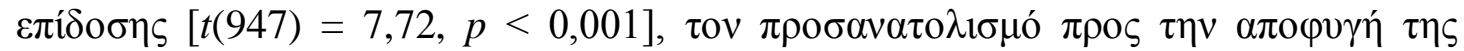

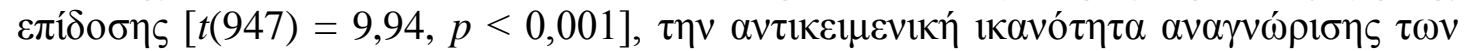

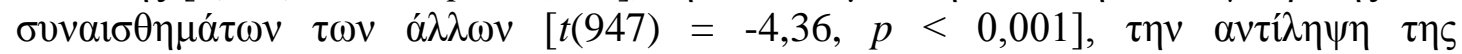

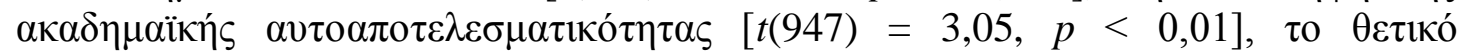

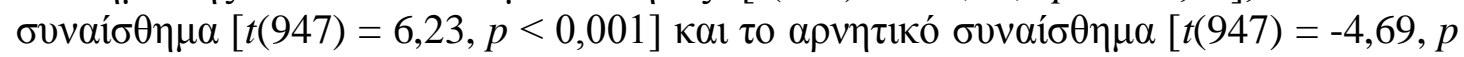

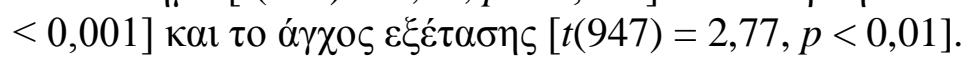

\section{$\Sigma v \zeta \dot{\eta} \tau \eta \sigma \eta$}

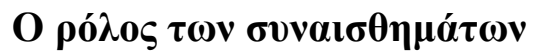

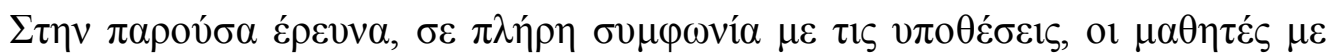

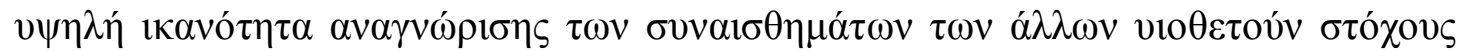

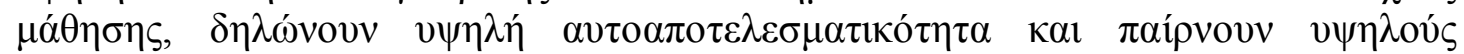

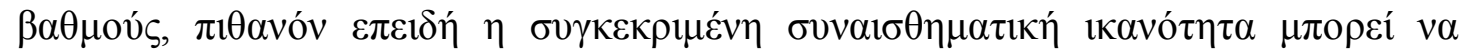

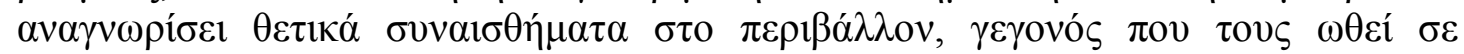

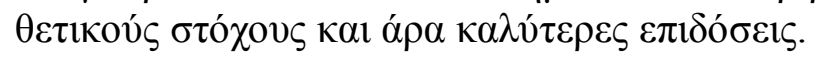

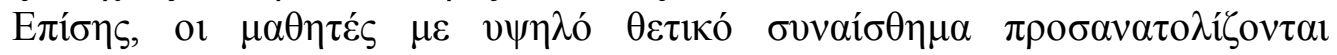

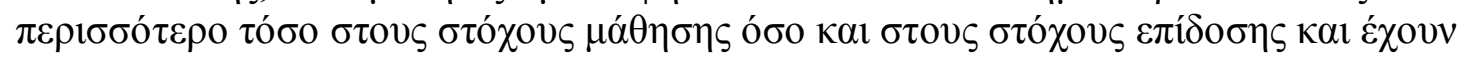

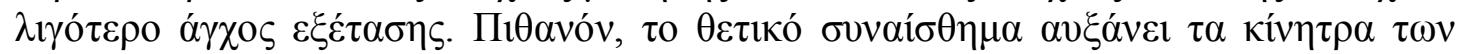

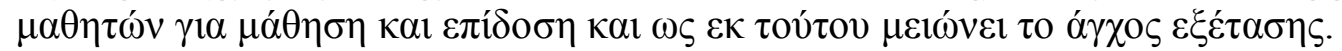

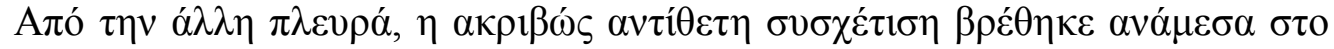

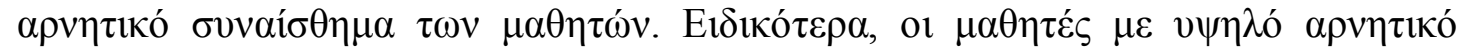

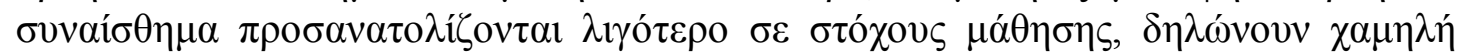

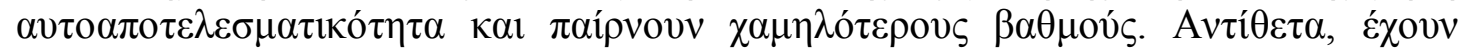

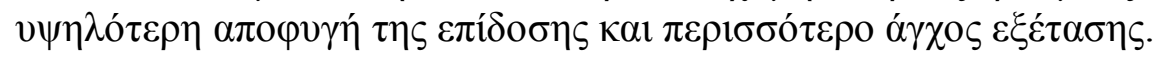

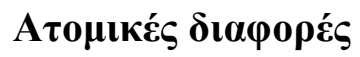

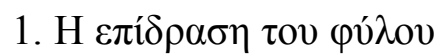

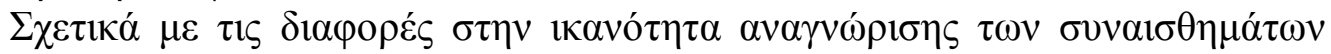

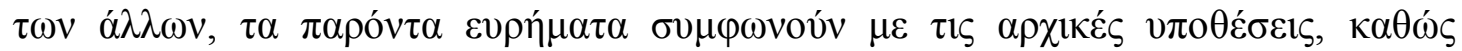

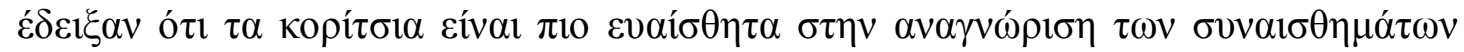




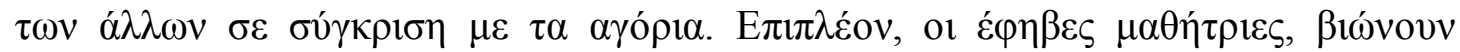

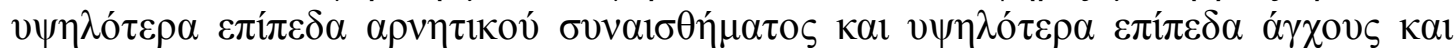

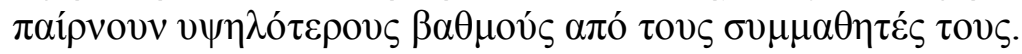

\section{2. $\mathrm{H} \varepsilon \pi i \delta \rho \alpha \sigma \eta \tau \eta \varsigma \eta \lambda \iota \kappa i ́ \varsigma$}

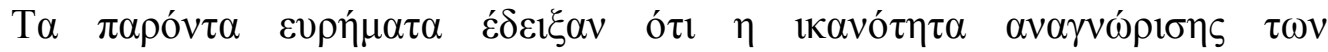

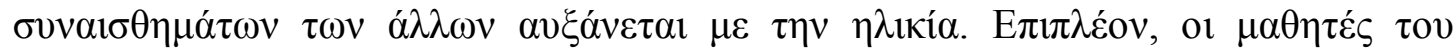

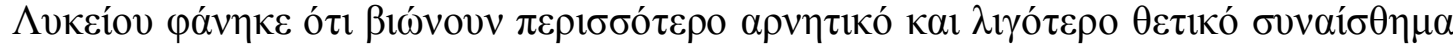

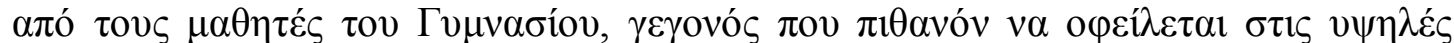

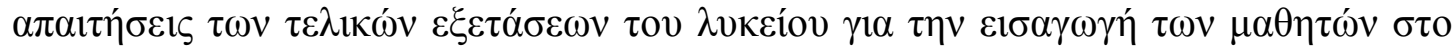

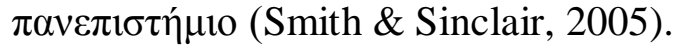

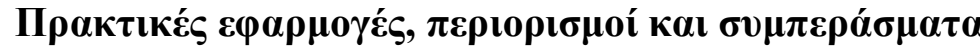

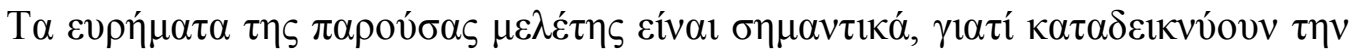

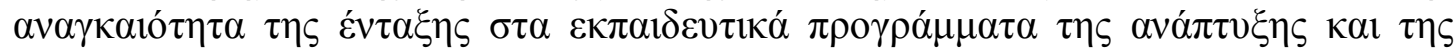

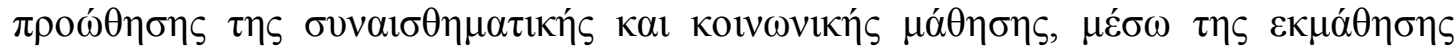

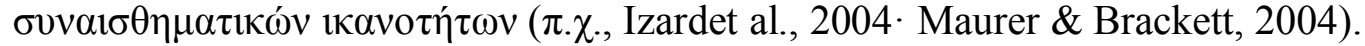

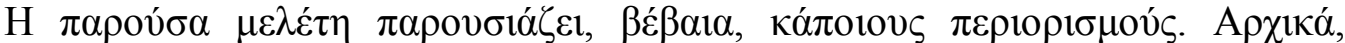

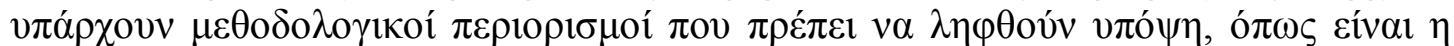

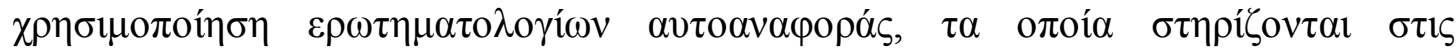

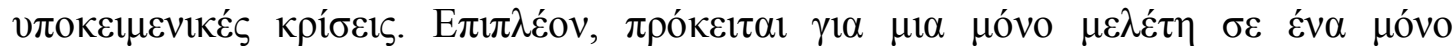

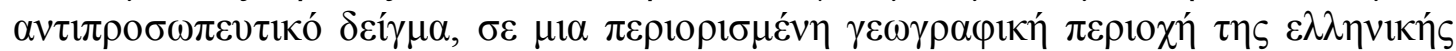

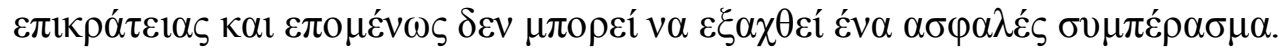

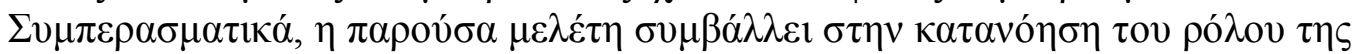

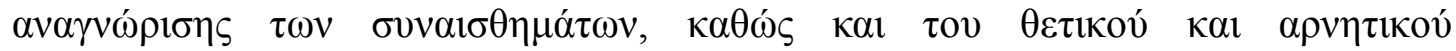

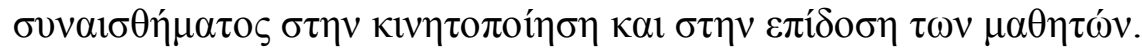

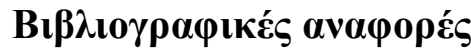

Anderman, E., \& Midgley C. (2002). Methods of studying goals, goal structures, and patterns of adaptive learning. In C. Midgley (Ed.), Goals, goal structures, and patterns of adaptive learning (pp. 1-20). Mahwah: Lawrence Erlbaum Associates.

Bandura, A. (1986). Social foundations of thought and action: A social cognitive theory. Englewood Cliffs, NJ: Prentice- Hall.

Biddle, S., Soos, I., \& Chatzisarantis, N. (1999). Predicting physical activity intentions using goal perspectives and self-determination theory approaches. European Psychologist, 4, 83-89.

Chan, D. W. (2005). Self-perceived creativity, family hardiness, and emotional intelligence of Chinese gifted students in Hong Kong. Journal of Secondary Gifted Education, 16, 47-56.

Cheng, H., \& Furnham, A. (2002). Personality, peer relations, and selfconfidence as predictors of happiness and loneliness. Journal of Adolescence, 25, $327-339$. 
Ciarrochi, J. V., Deane, F. P., \& Anderson, S. (2002). Emotional intelligence moderates the relationship between stress and mental health. Personality and Individual Differences, 32, 197-209.

Denham, S. A., Blair, K. A., DeMulder, E., Levitas, J., Sawyer, K., AuerbachMajor, S., Queenan, P. (2003). Preschool emotional competence: Pathway to social competence. Child Development, 74, 238-256.

Duda, J. L., \& Nicholls, J. G. (1992). Dimensions of achievement motivation in schoolwork and sport. Journal of Educational Psychology, 84, 290-299.

Dweck, C. S. (2000). Self-theories: Their role in motivation, personality, and development. Philadelphia, PA: Taylor \& Francis.

Dweck, C. S., \& Leggett, E. L. (1988). A social-cognitive approach to motivation and personality. Psychological Review, 95, 256-273.

Ekman, P. (2003). Emotions revealed: Recognizing faces and feelings to improve communication and emotional life. New York, NY: Times Books.

Hall, H. K., \& Kerr, A. W. (1997). Motivational antecedents of precompetitive anxiety in youth sport. The Sport Psychologist, 11(1), 24-42.

Hughes, C., \& Leekam, S. ( 2004 ). What are the links between theory of mind and social relations? Review, refl ections and new directions for studies of typical and atypical development. Social Development, 13, 590- 619.

Izard, C. E. (1989). The structure and functions of emotions: Implications for cognition, motivation, and personality. In I. S. Cohen (Ed.), The G. Stanley Hall lecture series (pp. 35-73). Washington, DC: American Psychological Association.

Kaplan, A., \& Maehr, M. L. (2007). The contributions and prospects of goal orientation theory. Educational Psychology Review, 19, 141-184.

Kavanagh, D. J., \& Bower, G. H. (1985). Mood and self-efficacy: Impact of joy and sadness on perceived capabilities. Cognitive Therapy and Research, 9, 507-525.

Linnenbrink, E. A., \& Pintrich, P. R. (2002). Achievement goal theory and affect: An asymmetrical bidirectional model. Educational Psychologist, 37, 69-78.

Mayer, J., Salovey, P., \& Caruso, D. R. (2000a). Models of emotional intelligence. In R. Sternberg (Ed.), Handbook of intelligence. New York: Cambridge University Press.

Mellanby, J., Martin, M., \& O'Doherty, J. (2000). The “gender gap' in final examination results at Oxford University. British Journal of Psychology, 91, 377-390.

Midgley, C., Maehr, M. L., Hicks, L., Roeser, R., Urdan, T., Anderman, E., Kaplan, A., Arunkumar, R., Middleton, M. (1997). Patterns of Adaptive Learning Survey (PALS). Ann Arbor, MI: Center for Leadership and Learning. 
Nowicki, S., \& Carton, E. (1997). The relation of nonverbal processing ability of faces and voices and children's feelings of depression and competence. Journal of Genetic Psychology, 158, 357-363.

Pekrun, R., Goetz, T., Titz, W, \& Perry, R P. (2002). Positive emotions in education. In E. Frydenberg (Ed.). Beyond coping: Meeting goals, visions, and Challenges (pp. 1 49- 1 74). Oxford, UK: Elsevier.

Pintrich, P. R., Smith, D. A. F., Garcia, T., \& McKeachie, W. J. (1991). A manual for the use of the motivated strategy for learning questionnaire (MSLQ). Ann Arbor, MI: National Centre for Research to Improve Post Secondary Teaching and Learning.

Sideridis, G. D. (2005). Goal orientation, academic achievement, and depression: Evidence in favor of a revised goal theory framework. Journal of Educational Psychology, 97, 366-375.

Smith, C.A., \& Sinclair, A.H. (2005) Molecular genetics of gonadal sex determination. In: Cytogenetics and Genome Research (Eds. Nanda, I., Schmid, M., Burt, D.), 109, 1-87.

Trockel, M. T., Barnes, M. D., \& Egget, D. L. (2000). Health-related variables and academic performance among first-year college students: Implications for sleep and other behaviors. Journal of American College Health, 49, 125-131.

Vassiou, A., Mouratidis, A., Andreou, E., \& Kafetsios, K. (2014, article in press). Students' achievement goals, emotion perception ability and affect and performance in the classroom: a multilevel examination. Educational Psychology.

Zembylas, M., \& Fendler, L. (2007). Reframing emotion in education through lenses of parrhesia and care of the self. Studies in Philosophy and Education, 26, 319333. 